\title{
Differential Feedback of Channel Gram Matrices for Block Diagonalized Multiuser MIMO Systems
}

\author{
Daniel Sacristán-Murga* and Antonio Pascual-Iserte*† \\ ${ }^{*}$ Centre Tecnològic de Telecomunicacions de Catalunya (CTTC), Spain \\ ${ }^{\dagger}$ Dept. of Signal Theory and Communications - Universitat Politècnica de Catalunya (UPC), Spain \\ Email: daniel.sacristan@cttc.es, antonio.pascual@upc.edu
}

\begin{abstract}
This paper proposes a differential quantization strategy to be used in the feedback link of a multi-input-multioutput (MIMO) multiuser broadcast communication system. This strategy is based on an algorithm presented for single user MIMO, and is applied to broadcast systems where the transmitter design is based on block diagonalization, i.e., the transmissions are optimized so as not to produce multiuser interference at the receive terminals, which are using more than one antenna each. The algorithm is applied to the channel Gram matrices exploiting geodesic curves and the intrinsic geometry of positive definite Hermitian matrices. Furthermore, the differential nature of the algorithm exploits the correlation in time present in real channels. Simulation results in the paper show that the proposed algorithm outperforms other techniques based on the direct quantization of the channel matrix.
\end{abstract}

Index Terms-MIMO systems, multiuser systems, broadcast channels, feedback communication, quantization, differential geometry.

\section{INTRODUCTION}

In MIMO broadcast (downlink) channels, dirty paper coding (DPC) has proved to achieve the capacity region [1], but it is very complex to implement and computationally intensive. Linear precoding techniques such as zero-forcing and block diagonalization (BD) [2] provide the same multiplexing gain as DPC, while incurring in an absolute loss in terms of throughput [3], [4], [5].

BD decomposes the overall MIMO broadcast channel (BC) of the different users into a set of parallel single-user MIMO channels without inter-user interference. In order to do this, accurate channel state information (CSI) is required at the transmitter. In scenarios where channel reciprocity does not hold, a feedback channel with limited capacity is used to send the CSI from the receiver to the transmitter. Because of the limited capacity of the feedback links and the impact that accurate CSI has on the design of the transmitter, proper quantization procedures to be applied to the channel estimates are of great importance.

The quantization and feedback strategy proposed in this paper is inspired by the one from [6] for the case of single-user MIMO. Its two defining characteristics are the quantization and feedback of the Gram of the channel matrix instead of the complete channel matrix, and the fact that it exploits the

This work was partially supported by the Catalan Government under grant 2009 SGR 891, by the Spanish Government under projects TEC2008-06327C03 (MULTI-ADAPTIVE) and 2A103 (MIMOWA) from MEDEA+ program (AVANZA I+D TSI-020400-2009-44), and by the European Commission under projects NEWCOM++ (216715) and BuNGee (248267). temporal correlation of the channel. The algorithm is based on a differential quantization over the cone of Hermitian, positive definite matrices using geodesic routes. [7] also used geodesic routes but its application was to the quantization of the subspace spanned by the eigenvectors of the channel matrix in the Grassmannian manifold. This constrains the power allocation between spatial transmission modes to be uniform, which degrades the performance. The strategy presented in this paper outperforms other feedback designs proposed recently in [8], [9] and [10], as will be shown with the help of simulations.

The paper is organized as follows. The system and signal models are described in section II. The differential algorithm based on geodesic routes for the case of single user MIMO is summarized in section III, while section IV contains the extension to the multiuser scenario. Section $\mathrm{V}$ provides numerical simulations of the different feedback strategies and section VI concludes the paper.

\section{SYSTEM AND SIGNAL MODELS}

We consider a MIMO broadcast system with a single transmitter and $K$ receivers or users, as depicted in Fig. 1. The transmitter has $n_{T}$ antennas and the $k$ th receiver has $n_{R k}$ antennas. The channel matrix from the transmitter to the $k$ th receiver is denoted by $\mathbf{H}_{k} \in \mathbb{C}^{n_{R k} \times n_{T}}$ and the associated precoding matrix by $\mathbf{B}_{k} \in \mathbb{C}^{n_{T} \times n_{S k}}$, where $n_{S k}$ is the number of streams to be transmitted to user $k . \mathbf{x}_{k} \in \mathbb{C}^{n_{S k}}$ represents the $n_{S k}$ streams of signals to be transmitted with $\mathbb{E}\left[\mathbf{x}_{k} \mathbf{x}_{k}^{H}\right]=\mathbf{I}$. The signal at the $k$ th receiver is thus:

$$
\begin{aligned}
\mathbf{y}_{k} & =\sum_{i=1}^{K} \mathbf{H}_{k} \mathbf{B}_{i} \mathbf{x}_{i}+\mathbf{w}_{k} \in \mathbb{C}^{n_{R k}} \\
& =\mathbf{H}_{k} \mathbf{B}_{k} \mathbf{x}_{k}+\mathbf{H}_{k} \tilde{\mathbf{B}}_{k} \tilde{\mathbf{x}}_{k}+\mathbf{w}_{k},
\end{aligned}
$$

where $\mathbf{w}_{k} \in \mathbb{C}^{n_{R}}$ is the AWGN at the receiver with $\mathbb{E}\left[\mathbf{w}_{k} \mathbf{w}_{k}^{H}\right]=\sigma_{w}^{2} \mathbf{I}$. $\tilde{\mathbf{B}}_{k}$ and $\tilde{\mathbf{x}}_{k}$ result, respectively, from the stack of the the precoding matrices and the transmit vectors for all users other than receiver $k$ :

$$
\begin{aligned}
\tilde{\mathbf{B}}_{k} & =\left[\begin{array}{llllll}
\mathbf{B}_{1} & \ldots & \mathbf{B}_{k-1} & \mathbf{B}_{k+1} & \ldots & \mathbf{B}_{K}
\end{array}\right], \\
\tilde{\mathbf{x}}_{k}^{T} & =\left[\begin{array}{llllll}
\mathbf{x}_{1}^{T} & \ldots & \mathbf{x}_{k-1}^{T} & \mathbf{x}_{k+1}^{T} & \ldots & \mathbf{x}_{K}^{T}
\end{array}\right] .
\end{aligned}
$$

The technique of BD [2] exploits the CSI in order to eliminate interference between users, i.e., the precoding matrices $\mathbf{B}_{k}$ are designed so that $\mathbf{H}_{i} \mathbf{B}_{j}=\mathbf{0}$ for $i \neq j$. The process is explained briefly in section IV. Consequently, the multiuser 


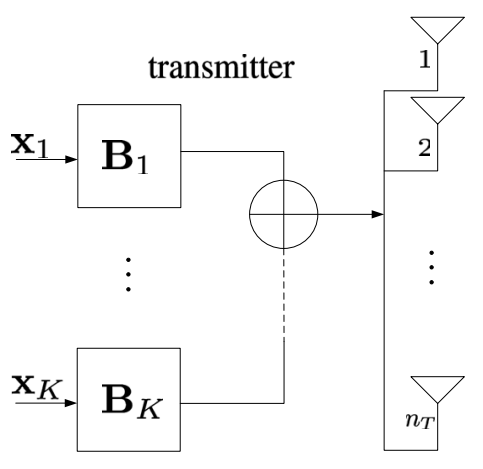

Fig. 1. System model.

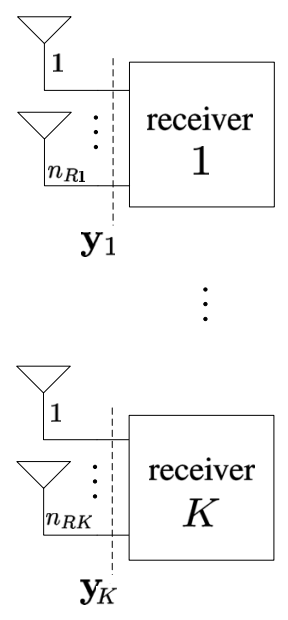

channel is divided into parallel single user MIMO channels, and (2) simplifies to:

$$
\mathbf{y}_{k}=\mathbf{H}_{k} \mathbf{B}_{k} \mathbf{x}_{k}+\mathbf{w}_{k}, k=1 \ldots K .
$$

Appropriate single user precoding techniques can then be used on top of the BD (for example, waterfilling over the channel eigenmodes to achieve capacity).

Single user MIMO techniques depend on accurate CSI in order to fully exploit the channel characteristics. In multiuser schemes the necessity for accurate CSI is even more extreme because, apart from a loss in each user performance, imperfect CSI produces inter-user interference (since in that case BD can not eliminate interference completely). This is more critical in the high SNR regime, precisely where multiuser MIMO shines most.

In this paper we assume that each receiver estimates perfectly its current channel matrix $\mathbf{H}_{k}$, and that the transmitter designs $\mathbf{B}_{k}$ assuming that the available CSI at its side is also perfect, i.e., without quantization or feedback errors. As will be shown in section IV, the CSI required at the transmitter for $\mathrm{BD}$ corresponds to the Gram matrix of the individual MIMO channels (i.e., $\mathbf{H}_{k}^{H} \mathbf{H}_{k}, k=1 \ldots K$ ).

\section{REVIEW OF THE FEEDBACK ALGORITHM FOR SINGLE USER MIMO}

In [6] we presented a feedback algorithm for single user MIMO based on the differential quantization of the channel Gram matrix $\mathbf{H}^{H} \mathbf{H}$. It has been proved that, for single user MIMO, the channel Gram matrix contains the sufficient information to design a transmitter that maximizes criteria such as signal to noise ratio (SNR), mutual information, or minimizes bit error rate (BER) or mean square error (MSE) [11]. This section summarizes the quantization algorithm for Gram matrices while section IV extends its application to multiuser MIMO systems using BD.

\section{A. Preliminaries on some differential geometry definitions}

The objective of the quantization is the channel Gram matrix, which is Hermitian and positive definite ${ }^{1}$. As shown

\footnotetext{
${ }^{1}$ The original work for single user assumed that the channel Gram matrix was strictly positive definite. For the multiuser case we will work with extended Gram matrices defined as $\widetilde{\mathbf{R}}_{H}=\mathbf{H}^{H} \mathbf{H}+\epsilon \mathbf{I}, \epsilon>0$, where $\epsilon$ is a predefined known constant, which are positive definite by construction.
}

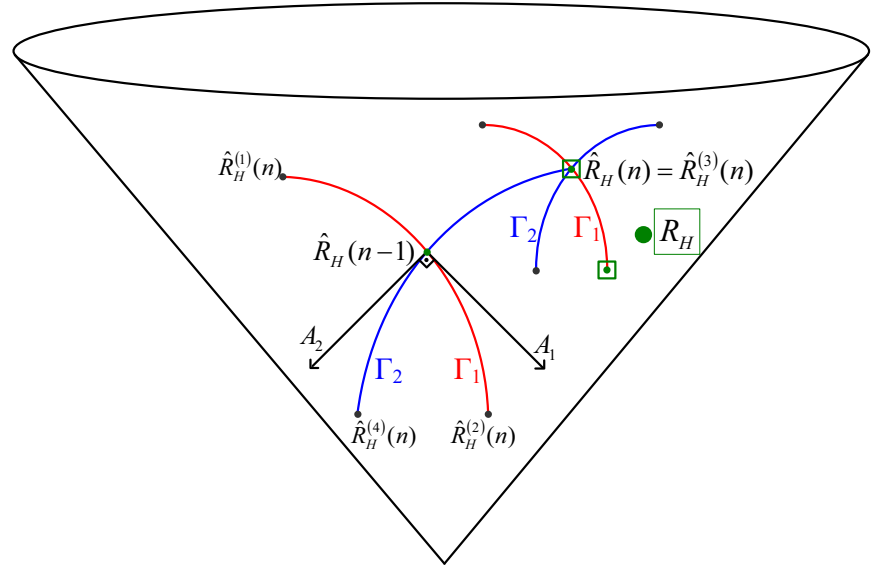

Fig. 2. 2-bit differential quantization in the space of channel correlation matrices.

in [12] the set of Hermitian positive definite matrices $\mathcal{S}=$ $\left\{\mathbf{R} \in \mathbb{C}^{n_{T} \times n_{T}}: \mathbf{R}^{H}=\mathbf{R}, \mathbf{R} \succ \mathbf{0}\right\}$ is a convex cone ${ }^{2}$, i.e., $\forall \mathbf{R}_{1}, \mathbf{R}_{2} \in \mathcal{S}, \forall s \geq 0, \mathbf{R}_{1}+s \mathbf{R}_{2} \in \mathcal{S}$. This set is described properly by the following definitions [12], [13]:

- Scalar product and norm: The scalar product between two Hermitian matrices $\mathbf{A}$ and $\mathbf{B}$ at any point $\mathbf{R}$ in this set $\mathcal{S}$ is defined as: $\langle\mathbf{A}, \mathbf{B}\rangle_{\mathbf{R}}=\operatorname{Tr}\left(\mathbf{R}^{-1} \mathbf{A} \mathbf{R}^{-1} \mathbf{B}\right)$.

- Geodesic curve: The geodesic curve $\Gamma(t)$ is the path that connects two points $\mathbf{R}_{1}$ and $\mathbf{R}_{2}$ in the set $\mathcal{S}$ with minimum distance and with all of its points in $\mathcal{S}$ :

$$
\Gamma(t)=\mathbf{R}_{1}^{1 / 2} \exp (t \mathbf{C}) \mathbf{R}_{1}^{1 / 2},
$$

where $\mathbf{C}=\log \left(\mathbf{R}_{1}^{-1 / 2} \mathbf{R}_{2} \mathbf{R}_{1}^{-1 / 2}\right), \Gamma(0)=\mathbf{R}_{1}$, and $\Gamma(1)=\mathbf{R}_{2}$. The direction of the curve at $t=0$ is the derivative at $t=0$, and is given by $\Gamma^{\prime}(0)=\mathbf{R}_{1}^{1 / 2} \mathbf{C R}_{1}^{1 / 2}$.

- Geodesic distance: The distance between any two points in $\mathcal{S}$ is given by the length of the geodesic curve that connects them. It is expressed as:

$$
\operatorname{dist}_{g}\left(\mathbf{R}_{1}, \mathbf{R}_{2}\right)=\left(\sum_{i}\left|\log \lambda_{i}\right|^{2}\right)^{1 / 2}
$$

where $\left\{\lambda_{i}\right\}$ are the eigenvalues of $\mathbf{R}_{1}^{-1 / 2} \mathbf{R}_{2} \mathbf{R}_{1}^{-1 / 2}$.

\section{B. Algorithm description}

The algorithm features a differential quantization of the channel Gram matrix $\mathbf{R}_{H}=\mathbf{H}^{H} \mathbf{H}$ instead of the whole channel matrix $\mathbf{H} . \mathbf{R}_{H}(n)$ represents the Gram of the channel matrix and $\mathbf{H}(n)$ the channel matrix at feedback instant $n$. The objective of the quantization is to minimize the geodesic distance ${ }^{3}$ between the actual channel Gram matrix $\mathbf{R}_{H}$ and its fed back estimate $\widehat{\mathbf{R}}_{H}$. The differential nature of the procedure allows to exploit the temporal correlation of the channel. The feedback algorithm is described in Table I.

\footnotetext{
${ }^{2}$ Actually, reference [12] is devoted to the case of real matrices, although the results and conclusions can be extended directly to the complex case.

${ }^{3}$ The algorithm from [6] considers the design criterion as the way to select the best quantization candidate. For the multiuser case, the minimum geodesic distance criterion is preferred, since the CSI is used to diagonalize the channel prior to incorporating the design criterion into the precoder.
} 
TABLE I

GRAM MATRIX GEODESIC QUANTIZATION AND FEEDBACK

Initialization: the algorithm starts from the cone vertex: $\widehat{\mathbf{R}}_{H}(0)=\mathbf{I}$.

1) The receiver and the transmitter generate a common set of $Q$ random Hermitian matrices using the same pseudo-random generator and the same seed, which is established by the design and introduces no additional complexity.

2) These matrices are then orthonormalized using the Gram-Schmidt procedure [14] according to the definition of scalar product presented in section III-A, producing the set $\left\{\mathbf{A}_{i}\right\}_{i=1}^{Q}$.

3) The receiver and the transmitter use $\left\{\mathbf{A}_{i}\right\}_{i=1}^{Q}$ to generate a set of $Q$ geodesic curves $\left\{\Gamma_{i}(t)\right\}_{i=1}^{Q}$ having all of them the same initial point $\mathbf{R}=\widehat{\mathbf{R}}_{H}(n-1)$ and with orthogonal directions: $\Gamma_{i}(t)=\widehat{\mathbf{R}}_{H}^{1 / 2}(n-1) \exp \left(t \mathbf{C}_{i}\right) \widehat{\mathbf{R}}_{H}^{1 / 2}(n-1)$.

The maximum number of orthogonal routes is given by the dimension of the set of Hermitian matrices, i.e., $Q \leq n_{T}^{2}$.

4) Each of these geodesic curves is used to generate two candidates for the feedback in the next iteration $\widehat{\mathbf{R}}_{H}(n)$, all of them equidistant to $\widehat{\mathbf{R}}_{H}(n-1)$. The distance is a design parameter related to the time correlation of the propagation channel. $\left\{\begin{aligned} \widehat{\mathbf{R}}_{H}^{(2 i-1)}(n) & =\Gamma_{i}(-1), & & 1 \leq i \leq Q, \\ \widehat{\mathbf{R}}_{H}^{(2 i)}(n) & =\Gamma_{i}(1), & & 1 \leq i \leq Q .\end{aligned}\right.$

5) Each candidate is evaluated at the receiver, and the one with the smallest geodesic distance to the actual $\mathbf{R}_{H}$ is selected. Its index $i_{F B}$ is sent to the transmitter through the feedback link.

6) The selected matrix will be used for the transmitter design and as the starting point in the next iteration:

$$
\begin{aligned}
i_{F B} & =\arg \min _{i} \operatorname{dist}_{g}\left(\widehat{\mathbf{R}}_{H}^{(i)}(n), \mathbf{R}_{H}(n)\right), 1 \leq i \leq 2 Q, \\
\widehat{\mathbf{R}}_{H}(n) & =\widehat{\mathbf{R}}_{H}^{\left(i_{F B}\right)}(n) .
\end{aligned}
$$

Fig. 2 shows the differential quantization process using 2 bits. Starting from $\widehat{\mathbf{R}}_{H}(n-1)$, the algorithm generates 2 geodesic routes $\Gamma_{1}(t)$ and $\Gamma_{2}(t)$ with orthogonal velocity matrices $\mathbf{A}_{1}$ and $\mathbf{A}_{2}$, respectively. The four quantization candidates are: $\widehat{\mathbf{R}}_{H}^{(1)}(n)=\Gamma_{1}(-1), \widehat{\mathbf{R}}_{H}^{(2)}(n)=\Gamma_{1}(1), \widehat{\mathbf{R}}_{H}^{(3)}(n)=\Gamma_{2}(-1)$, and $\widehat{\mathbf{R}}_{H}^{(4)}(n)=\Gamma_{2}(1)$. At the receiver, each candidate is compared to the actual $\mathbf{R}_{H}$ and the one with smallest geodesic distance to $\mathbf{R}_{H}$ (in this example candidate 3 ) is selected. That is, its index $i_{F B}=3$ is sent to the transmitter through the feedback channel and $\widehat{\mathbf{R}}_{H}(n)=\widehat{\mathbf{R}}_{H}^{(3)}(n)$. The next iteration starts from this point, generates 2 orthogonal routes and 4 quantization candidates, selects the candidate with the smallest geodesic distance to $\mathbf{R}_{H}$, and so on.

\section{EXTENSION TO MULTIUSER MiMO WiTH BlOCK DiAGONALIZATION}

\section{A. Introduction to Block Diagonalization:}

In order to remove all inter-user interference in the system presented in section II, the precoding matrices $\mathbf{B}_{k}$ are designed so that $\mathbf{H}_{i} \mathbf{B}_{j}=\mathbf{0}$ for $i \neq j$ [2]. This constraint forces $\mathbf{B}_{k}$ to lie in the null space of $\tilde{\mathbf{H}}_{k}$, with $\tilde{\mathbf{H}}_{k}$ defined as:

$$
\tilde{\mathbf{H}}_{k}=\left[\begin{array}{llllll}
\mathbf{H}_{1}^{T} & \ldots & \mathbf{H}_{k-1}^{T} & \mathbf{H}_{k+1}^{T} & \ldots & \mathbf{H}_{K}^{T}
\end{array}\right]^{T} .
$$

Note that the system dimension constraint is obtained from this definition. Block diagonalization as presented here is possible only if $n_{T}>\max \left\{\operatorname{rank}\left(\tilde{\mathbf{H}}_{1}\right), \ldots, \operatorname{rank}\left(\tilde{\mathbf{H}}_{K}\right)\right\}$.
An orthogonal basis for the null space of $\tilde{\mathbf{H}}_{k}$ can be obtained as the right singular vectors of $\tilde{\mathbf{H}}_{k}$ corresponding to the zero singular values. Then, a matrix $\tilde{\mathbf{V}}_{k}^{(0)} \in$ $\mathbb{C}^{n_{T} \times\left(n_{T}-\operatorname{rank}\left\{\tilde{\mathbf{H}}_{k}\right\}\right)}$ can be defined containing as columns such singular vectors of $\tilde{\mathbf{H}}_{k}$. These are equal to the eigenvectors corresponding to the zero eigenvalues of $\tilde{\mathbf{H}}_{k}^{H} \tilde{\mathbf{H}}_{k}$, which is obtained from the feedback scheme presented in this paper. From (8), it follows directly that $\tilde{\mathbf{H}}_{k}^{H} \tilde{\mathbf{H}}_{k}$ can be computed at the transmitter as the sum of the individual channel Gram matrices corresponding to users different from $k$ :

$$
\tilde{\mathbf{H}}_{k}^{H} \tilde{\mathbf{H}}_{k}=\sum_{i=1, i \neq k}^{K} \mathbf{R}_{H i},
$$

where $\mathbf{R}_{H i}=\mathbf{H}_{i}^{H} \mathbf{H}_{i}$, i.e., by means of the feedback of matrices $\mathbf{R}_{H i}$, matrix $\tilde{\mathbf{H}}_{k}^{H} \tilde{\mathbf{H}}_{k}$ can be computed.

The interference elimination is achieved through multiplying at the transmitter by the correspondent $\tilde{\mathbf{V}}_{k}^{(0)}$, i.e., the channel is transformed to $\mathbf{H}_{k}^{\prime}=\mathbf{H}_{k} \tilde{\mathbf{V}}_{k}^{(0)}$. This scheme divides the multiuser channel into parallel single user MIMO channels. The transmitter then has to send a second sounding sequence through the parallelized channels so that the receivers can estimate the "new" channels $\mathbf{H}_{k}^{\prime}$. On top of the parallelized channels, any single user MIMO scheme can be implemented 4 through a precoder $\mathbf{M}_{k}$, for example waterfilling over the parallelized channel eigenmodes to achieve capacity. For this particular case the eigenvalues $\boldsymbol{\Sigma}_{k}$ and their associated eigenvectors $\mathbf{V}_{k}^{(1)}$ are computed for each of the channel Gram matrices $\mathbf{H}_{k}^{\prime}{ }_{k} \mathbf{H}_{k}^{\prime}$. The optimal power allocation coefficients $\boldsymbol{\Lambda}_{k}$ are then found using water-filling on $\boldsymbol{\Sigma}_{k}$, assuming a total power constraint $P_{k}$ for user $k$ [15]. Finally the precoding matrix $\mathbf{B}_{k}$ is set as:

$$
\mathbf{B}_{k}=\tilde{\mathbf{V}}_{k}^{(0)} \mathbf{M}_{k}, \mathbf{M}_{k}=\mathbf{V}_{k}^{(1)} \boldsymbol{\Lambda}_{k}^{1 / 2}
$$

The term $\tilde{\mathbf{V}}_{k}^{(0)}$ ensures that there is no multiuser interference and $\mathbf{V}_{k}^{(1)} \boldsymbol{\Lambda}_{k}^{1 / 2}$ is the optimum design satisfying the previous constraint. This example considered the design that achieves capacity but other design criteria can also be used [11].

\section{B. Feedback of the Gram matrix:}

As shown in the previous section, the transmitter can eliminate multiuser interference with knowledge of the channel Gram matrices of the individual receivers, i.e., it is not necessary to have the whole channel matrix; only its Grammian is required. Furthermore, once the interference has been removed, single user MIMO precoder designs can be applied. The optimum transmitter design for most interesting criteria such as the maximization of the mutual information or the SNR, or the minimization of the BER or the MSE, has been shown to depend also on the channel Gram matrix rather than on the complete channel matrix [11].

Consequently, by implementing the feedback of the channel Gram matrix from section III it is possible to first eliminate multiuser interference through BD and then compute the precoder designs. A summary of the BD with geodesic feedback of the Gram matrices is given in Table II.

\footnotetext{
${ }^{4}$ Note that the power constraint in the design of the precoder $\mathbf{M}_{k}$ does not change since $\tilde{\mathbf{V}}_{k}^{(0)}$ is a unitary transformation.
} 
TABLE II

BLOCK DIAGONALIZATION WITH GRAM MATRIX FEEDBACK

1) Receiver $k$ estimates $\mathbf{H}_{k}$ and sends $\mathbf{R}_{H k}$ through the feedback link.

2) At the transmitter, compute $\tilde{\mathbf{H}}_{k}^{H} \tilde{\mathbf{H}}_{k}=\sum_{i=1, i \neq k}^{K} \mathbf{R}_{H i}$.

3) At the transmitter, compute $\tilde{\mathbf{V}}_{k}^{(0)}$, the right null space of $\tilde{\mathbf{H}}_{k}^{H} \tilde{\mathbf{H}}_{k}$.

The parallelized channels are then $\mathbf{H}_{k}^{\prime}=\mathbf{H}_{k} \tilde{\mathbf{V}}_{k}^{(0)}$, and the transmitter knows $\mathbf{H}_{k}^{\prime}{ }^{H} \mathbf{H}_{k}^{\prime}=\tilde{\mathbf{V}}_{k}^{(0) H} \mathbf{R}_{H k} \tilde{\mathbf{V}}_{k}^{(0)}$.

4) Send a second sounding signal so that the receiver can estimate $\mathbf{H}_{k}^{\prime}$.

5) At the transmitter, design a precoder $\mathbf{M}_{k}$ for each of the parallel

channels $\mathbf{H}_{k}^{\prime}$ with the given design criterion using $\mathbf{H}_{k}^{\prime} H \mathbf{H}_{k}^{\prime}$.

6) Set the precoding matrices as: $\mathbf{B}_{k}=\tilde{\mathbf{V}}_{k}^{(0)} \mathbf{M}_{k}$.

\section{Simulation RESUlTS}

In the simulations, we consider a random multiuser MIMO channel with 4 antennas at the transmitter and 2 receivers with 2 antennas each. The time correlation of the channel is generated following a first order auto-regressive time-variation model according to the expression:

$$
\mathbf{H}(n)=\rho \mathbf{H}(n-1)+\sqrt{1-\rho^{2}} \mathbf{N}(n),
$$

where matrices $\mathbf{H}(n-1)$ and $\mathbf{N}(n)$ are assumed to be independent and composed of i.i.d. zero-mean complex Gaussian entries with unit variance. The time correlation factor $\rho$ models the variability of the channel and depends on the Doppler frequency of the terminal $f_{D}$ through the expression $\rho=J_{0}\left(2 \pi f_{D} \tau\right)$ [16], where $J_{0}$ is the zeroth-order Bessel function of the first kind and $\tau$ is the time difference between consecutive feedback instants. The case of a constant channel corresponds to $\rho=1$.

The first 2 simulations (Fig. 3 and Fig. 4) show the achievable sum-rate of different feedback strategies versus elapsed time, for a constant and a time variant channel ( $\rho=0.999)$, respectively. It can be seen how the proposed algorithm exploits the temporal correlation of the channel to improve progressively the accuracy of the feedback. For these simulations the transmitted power is fixed to $20 \mathrm{~dB}$ more than the channel noise. The algorithm presented in this paper is compared to a system with full cooperation between receivers (which is an upper bound to the performance, as it is equivalent to the single-user system with 4 receive antennas), to the case of block diagonalization with perfect CSI, a differential strategy based on a differential pulse code modulation (DPCM) quantization used in [9] and a strategy based on nondifferential uniform quantization of the channel Gram matrix coefficients presented in [8]. The plot shows how the proposed algorithm using only 4 bits of feedback performs similar to [9] with 16 bits of feedback. The differential algorithms clearly outperform the non-differential ones after few time instants, even when using 45 bits in the non-differential scheme (this applies also to other non-differential algorithms such as the one presented in [10]).

Fig. 5 shows the achievable sum-rate as a function of the SNR, defined as the ratio between the transmitted power and the noise power. Special interest should be paid to the mid and high SNR regime, because it is where multiuser MIMO provides the largest performance gain. In order to take into account the fact that the algorithm exploits the correlation

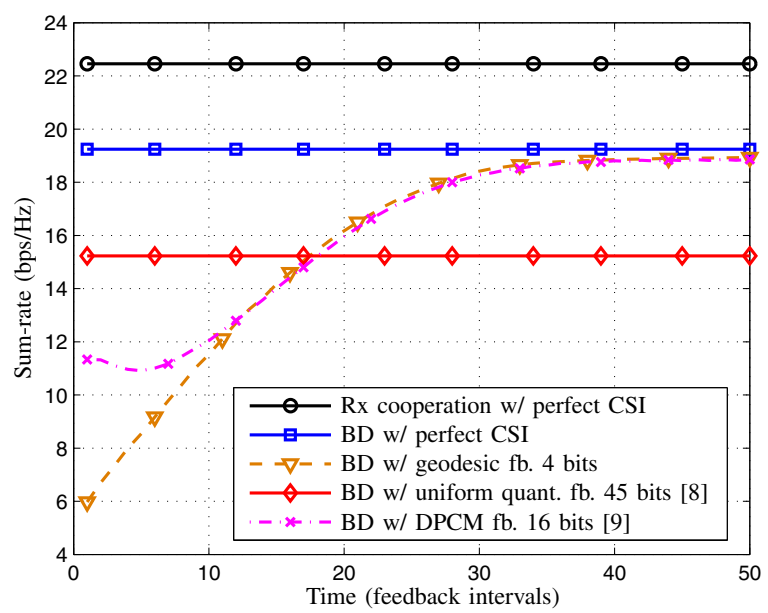

Fig. 3. Achievable sum-rate in a constant $(\rho=1)\{2,2\} \times 4$ system.

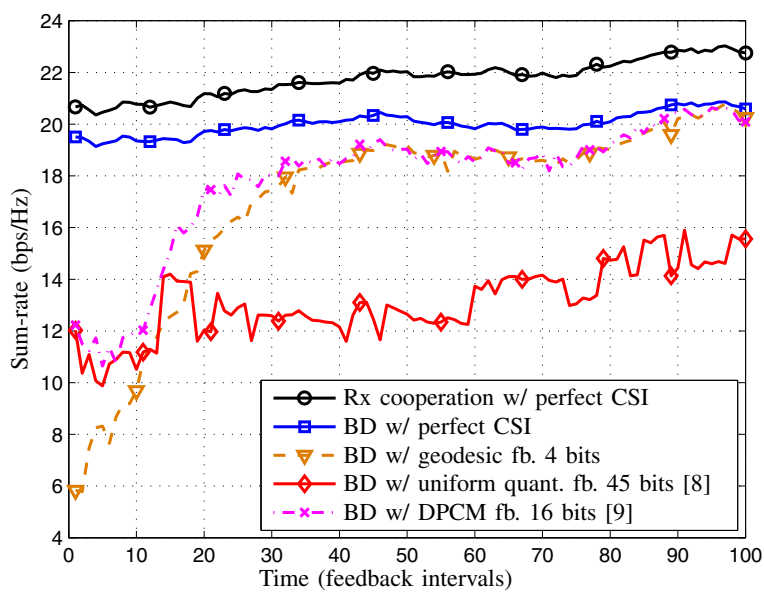

Fig. 4. Achievable sum-rate in a time-variant $(\rho=0.999)\{2,2\} \times 4$ system.

in time of the channel, the performance is compared after 30 time intervals. The case of full cooperation between the receivers is an upper bound to the performance and is depicted for comparison reasons. The simulations show how BD with perfect CSI achieves the maximum multiplexing gain, although an absolute difference in terms of throughput exists when compared to full receive cooperation. The algorithm presented here clearly provides a better sum-rate than the non-differential algorithm from [8]. As the transmission power increases, the accuracy of the CSI is more important, since the system is interference-limited and small inaccuracies in the channel estimate at the transmitter introduce a fixed amount of interference that produces a ceiling in the throughput of the system.

Regarding the signal to interference plus noise ratio (SINR) at the receivers, Fig. 6 shows the result of a simulation using different techniques. For this simulation the transmitted power is distributed evenly among the users, $\sigma_{w}^{2}=1$, and the results are averaged over 3000 channel realizations. The performance of the geodesic feedback algorithm corresponds to the instant after 30 feedback intervals in a constant channel. This simulation shows that the algorithm presented in this paper performs better than the other feedback strategy, even with less bits of feedback.

Finally, the bit error rate (BER) using a BPSK modulation is 


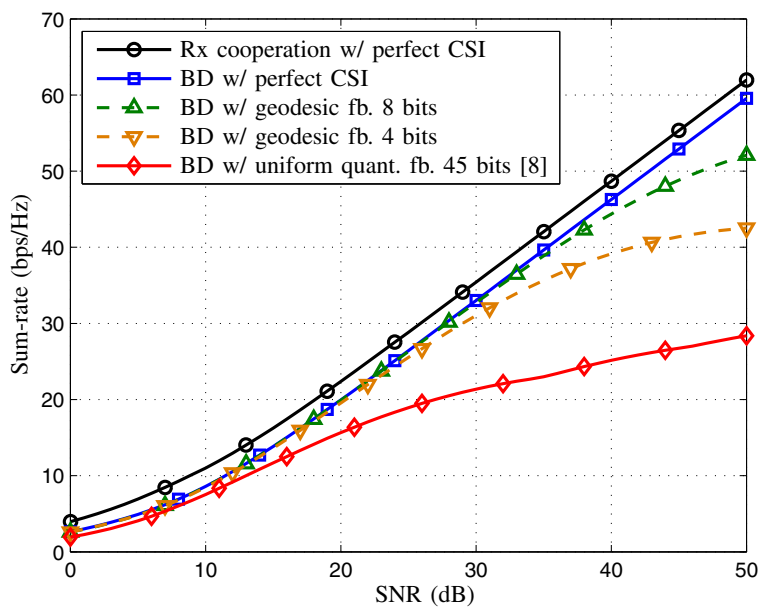

Fig. 5. Achievable sum-rate versus $S N R$ in a $\{2,2\} \times 4$ system.

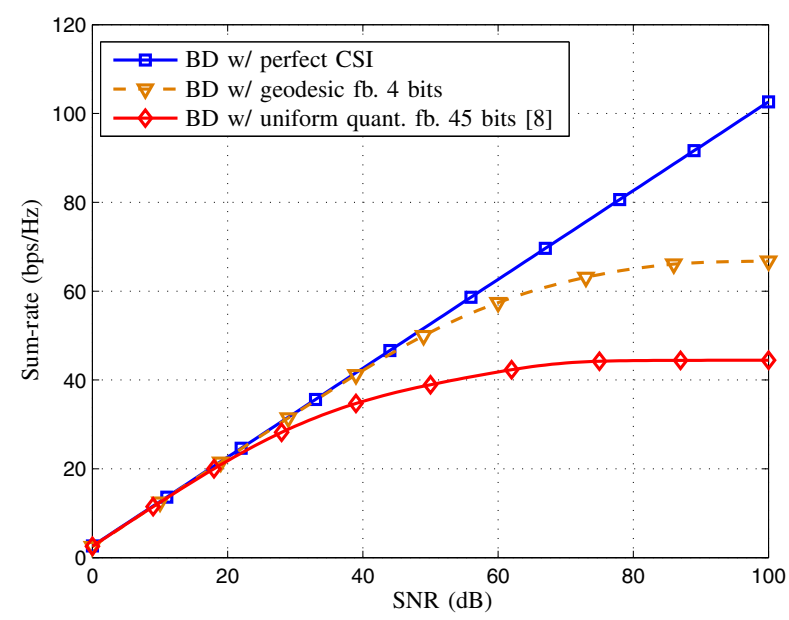

Fig. 6. Receiver SINR vs. SNR in a $\{2,2\} \times 4$ system.

simulated and the results after 30 time intervals are shown in Fig. 7. The transmission power is distributed evenly among the users, and the results are averaged over 3000 channel realizations. The figure shows that the proposed algorithm achieves a lower BER than the feedback strategies from [8] and requires less bits of feedback.

\section{Conclusions}

The feedback strategy proposed for MIMO multiuser broadcast systems that are based on BD has several advantages over other existing feedback techniques.

First, it considers exclusively the CSI that is absolutely required for optimum $\mathrm{BD}$ and precoding designs. Techniques based on quantization of the whole channel matrix are suboptimum because, as has been proved, only the Gram of the channel matrix is required at the transmitter. Furthermore, the quantization is performed over the cone of positive Hermitian matrices, exploiting the geometry of the domain space using geodesic curves.

Secondly, the differential nature of the algorithm exploits the correlation in time present in most channels to progressively refine the accuracy of the feedback.

Simulations show that this strategy achieves better performance than other techniques based on direct quantization of

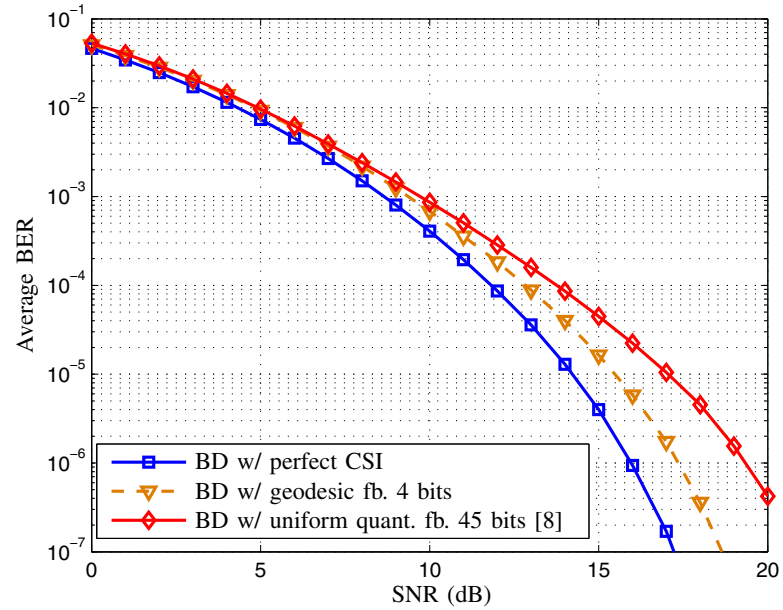

Fig. 7. BER vs. SNR in a $\{2,2\} \times 4$ system.

the coefficients of the Gram matrix.

\section{REFERENCES}

[1] H. Weingarten, Y. Steinberg, and S. Shamai, "The capacity region of the Gaussian multiple-input multiple-output broadcast channel," IEEE Trans. on Information Theory, vol. 52, no. 9, pp. 3936-3964, Sep. 2006.

[2] Q. H. Spencer, A. L. Swindlehurst, and M. Haardt, "Zero-forcing methods for downlink spatial multiplexing in multiuser MIMO channels," IEEE Trans. on Signal Processing, vol. 52, no. 2, pp. 461-471, Feb. 2004.

[3] Z. Shen, R. Chen, J. G. Andrews, R. W. Heath, and B. L. Evans, "Sum capacity of multiuser MIMO broadcast channels with block diagonalization," IEEE Trans. on Wireless Communications, vol. 6, no. 6, pp. 2040-2045, Jun. 2007.

[4] J. Lee and N. Jindal, "High SNR analysis for MIMO broadcast channels: dirty paper coding versus linear precoding," IEEE Trans. on Information Theory, vol. 52, no. 12, pp. 4787-4792, Dec. 2007.

[5] R. Hunger and M. Johan, "On the asymptotic optimality of blockdiagonalization for the MIMO BC under linear filtering," in Proc. International ITG Workshop on Smart Antennas, Feb. 2009.

[6] D. Sacristán-Murga and A. Pascual-Iserte, "Differential feedback of MIMO channel correlation matrices based on geodesic curves," in Proc. IEEE ICASSP'09, Apr. 2009.

[7] T. Pande, D. J. Love, and J. V. Krogmeier, "Reduced feedback MIMOOFDM precoding and antenna selection," IEEE Trans. on Signal Processing, vol. 55, no. 5, pp. 2284-2293, May 2007.

[8] C.-B. Chae, D. Mazzarese, N. Jindal, and R. W. Heath, "Coordinated beamforming with limited feedback in the MIMO broadcast channel," IEEE Journal on Selected Areas in Communications, vol. 26, no. 8, pp. $1505-1515$, Oct. 2008

[9] Y. Xiao, W. Miao, M. Zhao, S. Zhou, and J. Wang, "Limited-feedback modified block diagonalization for multiuser MIMO downlink with timevarying channels," in Proc. IEEE ICC'09, Jun. 2009.

[10] N. Ravindran and N. Jindal, "Limited feedback-based block diagonalization for the MIMO broadcast channel," IEEE Journal on Selected Areas in Communications, vol. 26, no. 8, pp. 1473-1482, Oct. 2008.

[11] D. P. Palomar, J. M. Cioffi, and M. A. Lagunas, "Joint Tx-Rx beamforming design for multicarrier MIMO channels: a unified framework for convex optimization," IEEE Trans. on Signal Processing, vol. 51, no. 9 , pp. 2381-2401, Sep. 2003.

[12] M. Talih, "Geodesic Markov chains on covariance matrices," Statistical and Applied Mathematical Sciences Institute, Tech. Rep., Mar. 2007.

[13] X. Pennec, P. Fillard, and N. Ayache, "A Riemannian framework for tensor computing," International Journal of Computer Vision, vol. 66, no. 1, pp. 41-66, Jan. 2006, a preliminary version appeared as INRIA Research Report 5255, July 2004.

[14] G. H. Golub and C. F. Van Loan, Matrix Computations, 3rd ed. The Johns Hopkins University Press, 1996.

[15] T. M. Cover and J. A. Thomas, Elements of Information Theory. WileyInterscience, 2006.

[16] R. Steele and L. Hanzo, Eds., Mobile Radio Communications, 2nd ed. John Wiley \& Sons, 1999. 